\title{
Geographic Data Informs Funding and Management of Metro Bike Share System
}

\author{
Pamela J. Schmidt \\ Washburn University, USA \\ pamela.schmidt@washburn.edu
}

\author{
Jia Feng \\ University of Nevada, Reno, USA \\ jiaf@unr.edu
}

\author{
Ronald Freeze \\ University of Arkansas, USA \\ rfreeze@uark.edu
}

\begin{abstract}
Geographic data is often used to supplement business data, but geographic location (GeoLocation) data has business value in its own right. This geospatial study presents a midwestern town's use of location analytics to infer the purpose for bike trips (usage purpose) and perform what-if analysis to enhance transportation options. The study applies spatial data analysis of bikeshare within transit management and public planning to address funding sources and public good. This case includes GeoExtension of the Metro Bike Share source data by utilizing U.S. Census data. The overall Metro Transit operational goal is to effectively manage the rideshare program to maximize community benefits, particularly in offering optimal transit options. Analysis to inform business operations are 1) inferring likely purpose for bike rides to differentiate between transportation and leisure; 2) determine if bike use integrates with other transit offerings, and 3) to provide transportation options to residents in low-income areas.
\end{abstract}

\section{Introduction}

\subsection{Sharing Economy and Transportation}

The rapidly expanding "sharing economy" addresses the efficient use of resources to address the needs of communities, particularly cities that deal with population growth, increasing density [1] and economic disparities [2]. A major challenge of transportation services is to cost-effectively operate a public transportation system that meets the needs of a diverse mobile society, particularly the needs of the economically disadvantaged. Bike ride sharing programs have shown substantial growth in the last decade, particularly in Europe and the USA [1]. There are many reasons why riders may choose to ride using a community-based rideshare system. A survey of rider intentions shows the top reasons include: 1) prefer the experience of cycling, 2) faster and more convenient than public transportation, 3 ) better for the environment and 4) use in addition to public transportation or 5) close to origin/destination [2]. A study in Washington D.C. showed that riders were motivated by savings in travel time ( $73 \%$ of users) and cost (25\% of users) [3].

Communities strive to operate public transportation to meet community needs but must also manage such services economically within funding constraints. The European Union (EU) Parliament's study identifies Bikesharing first in its list of five forms of shared mobility and points out the challenge to ensure financial stability of the transportation ecosystem as an indispensable underlying infrastructure [6]. This report points out that the EU shared mobility overall market in 2015 was estimated at 5.1 billion EUR, with an estimate of transactions in 2025 above 100 billion EUR. In the USA, a bikeshare equity report [6] exposes a gap in addressing social and economic inequalities in most cities' bikeshare programs. This USA report calls for planning agencies and local governments to address these inequities. Corrective actions suggest funding for public subsidies addressing low income areas and providing educational resources [6].

With the embedded GPS units in the shared bikes, bike trip data have gained popularity in urban planning, transportation geography and policy, gender studies, and travel behavior studies. Some projects focus on the travel pattern, land use and gender equality analysis [4] while others look at the bike share programs from an urban planning and policy perspective [5] [6]. A gap in the sharing economic literature is to address funding support, effective business management of allocated funds, as well as to offer equitable service to the community - especially service to the economically disadvantaged, to whom public transportation is a critical need. Focusing on the Topeka Metro Bike Share (MBS) program, we try to examine the business applications and policies using the GPS location information embedded in the shared bikes from 2017 and 2019. 


\subsection{Metro Bike Management Questions}

In this exploratory analysis, we focus on three business research questions: (1) what rides are most likely to be for transportation purposes versus those most likely for leisure or recreation, (2) how closely could the bike program connect with and extend the Metro bus transit system, and (3) the extent to which the MBS services have been utilized by the lowincome communities in the city?

\section{Geo-Spatial Data Uses}

Geographic data, in the form of a time-based GPS latitude and longitude location, provides rich representations that can be used for tracking assets and tracking metered use of assets. This study of one metropolitan area's bike ride share helps inform management and guide decision making to benefit the metro area transportation system. Being an important element in the global information infrastructure, GPS provides services in many industries, such as the location services on smart phones, watches, truck transportation, and global shipping services. In academic applications, the GPS locational service is essential in various fields. In farming, GPS is well applied to monitor and promote precision agriculture [16] [17]. In biology, GPS tracking services provide data for inferring animal behavior and understanding animal habitat [18] [19]. In environmental studies, GPS services can be used to monitor ocean shore/forest/glacier changes [20] [21] [22] [23]. In social studies, GPS technology aids in understanding behavior and environmental exposure [18] [19]. The usage and impact of GPS-enabled information has increased and it can be argued that the GPS adoption could enhance the information quality and the decision-making process.

For the rest of this paper, we introduce the Metro Bike System followed by our analysis of the three managerial questions. Post hoc data analysis informs management by addressing three questions: 1) what the likely purpose for bike rides is (differentiate between transportation and recreation), 2) whether the bike use serves to extend the mass transit bus offerings, and 3) whether rideshare is a useful transportation option to residents in low-income areas. This later analysis uses open data from the U. S. Census Bureau [27] to geographically overlay and

\footnotetext{
1 The Data Dictionary for data used in this analysis is available on request.
}

consider social economic factors related to the Metro Bike system layout and use.

\section{Metro Bike Share System}

As the first bike share program in Kansas, the Metro Bike Share (MBS) program started in the state capitol of Topeka in April 2015 with 100 bikes and a single full-time employee. Growing to 200 bikes for local community use within 2 years, the MBS program uses GPS devices on each bike to collect the locational information to assist the program management. As the service matured, the MBS director sought to improve their service by using GPS and other data, to understand bike ride characteristics, to explore possible connectivity between bus ridership and bike rides, and to examine the relationship between community characteristics and the bike ride behaviors.

The service provided in this study uses special high reliability, GPS-technology-enabled bikes, an online technology system for the Metro Bike administration and three user-interface options for riders (on bike interface, mobile app, and webpage interface). The method for managing the service is by pre-defined summary status screens with a high-level view of primary service elements such as: 1) rider members, 2) bike usage and 3) pre-defined summary status reports. For greater understanding of actual bike service usage, the website provides the ability to export raw data in the form of .csv files ${ }^{1}$. The use of the exported detailed data files supports the ability to do operational analysis needed by MBS management. Further, it supports the ability to augment analysis with publicly open data sources such as the U.S. Census and the weather data.

Inevitably, locational data have fundamental characteristics that are native to the GPS data collection process. The locational accuracy should be considered since the recreation-grade GPS units usually have a locational accuracy over several meters. Also, since the GPS locations are recorded in predetermined time intervals, the recorded start and end locations for each trip might be slightly different from the actual start and end locations. Further, it is important to acknowledge that trips generated from GPS location data might not be accurate since curved lines are straightened based on the recorded GPS locations on a certain time interval. The following analyses illustrate how the inclusion of the locational 
data and spatial data analysis system, in this case ESRI ArcGIS Pro $^{2}$, enrich MBS business data analysis and inform system usage.

As studied in this paper, the primary goal of Metro Ride Share's management is to understand the degree to which ride share is used for transportation such as work, errands and other economic activities versus leisure, exercise and recreation usage. As the city transit funding source has specific goals of benefiting public transit, this issue relates to targeted use of fund allocations. As the community likely benefits from Bike Share in many ways, this analysis could support seeking funding to address goals related to recreation, leisure and tourism activities. Also, alternate funding could be sought for intangible benefits such as enhancing community profile as livable, healthy, and concerned about climate change.

A key MBS management need is how to evaluate ridership based on data routinely collected by the ride share technology, and to do analysis quickly on the data collected. One slow, laborious alternative is to map out the GPS path of each ride, study maps and attempt to infer purpose of ride based on studying the mapped GPS path detail for each ride. Other alternatives considered were ride speeds and time/day of the week.

\section{Research Methods}

In the MBS program, the bike GPS system provides a practical way to track the locational information for each bike in the system at a 15-second time interval. This supports a closer look at the trip generation, trip duration, trip length and ending locations between 2017 and 2019. To enhance conventional business analysis, we incorporated spatial data analysis using the ESRI ArcGIS Desktop and ESRI ArcGIS Pro systems to answer our three research questions stated earlier. With an interest to connect the bike location information and other publicly available data, we adopt various spatial data analysis tools to complete our tasks.

\subsection{Data}

This analysis uses two years of the MBS locational data collected between October 1, 2017 and September 30, 2019. Each bike ride is recorded with the trip ID, user ID, time, duration, trip distance, and

\footnotetext{
2 Maps in this publication were created using ArcGIS® software by Esri. ArcGIS $®$ and $\operatorname{ArcMap}^{\mathrm{TM}}$ are the intellectual property of Esri and are used herein under license. Copyright $(\subset$ Esri.
}

locational information approximately every $10-15$ seconds. Census data for a given ride's start or stop locations act as a proxy for user characteristics. Locations for the Metro bus stops are adopted to supplement the MBS trip data for the transit extension analysis.

The data in this study are collected from four different sources. (1) The bike location data and user information are obtained from the Topeka Metro Bike Service (MBS). The data includes the basic information for the bike users including user bike ride distance, ride time, rider demographics and calculated calorie information. While the bike location data are collected directly from the bikes, a total of 149 bike rack locations are provided by the Metro Bike Service program. (2) The location information of a total of 590 bus stops are obtained from the Topeka Metro Services. (3) The Median Household Income information is from the U.S. Census Bureau. We assess the data in two ways. First, the ArcGIS Pro has the Enrich function to extract the Census Data such as Median Household Income (MHI) and Per Capita Income (PCI) based on an aggregated region around point locations. We used this function to aggregate the MHI and PCI information for each bike trip starting location with the hypothesis that the aggregated MHI/PCI information reflects bike user's socioeconomic status. The second way we access the average city MHI and PCI is directly from the American Community Survey published by the Census Bureau. (4) The World Topographic map provided by the ArcGIS Pro Basemap service is used to provide a locational reference.

\subsection{Methods Design}

The bike share studies across different fields adopt various methods depending on the research objectives. Trip time, station information, trip density, user demographics, zoning, and users' intentions are all important indicators in understanding the bikeshare projects in different cities in the world [4] [6] [28]. In this study, we focus mainly on the bike trip information and use the starting/ending locations as a proximal reference to understand the bike trip characteristics. To address the research questions, we utilized spatial data analysis tools using the ESRI ArcGIS Pro system.

All rights reserved. For more information about Esri® software, please visit www.esri.com. 


\section{Analysis}

This analysis uses the locational data collected by over 200 shared bikes between October 1, 2017 and September 30, 2019. Each bike ride is recorded with the trip ID, user ID, starting time, duration, trip distance, and locational information at a 15 -second time interval. In addition, the user characteristics and bus stop information are adopted to supplement the trip data. With the data stored on the Walton College Teradata server at the University of Arkansas ${ }^{3}$, we use ArcGIS Pro to access the data remotely. In this exploratory study, we focus on three business research questions: (1) can we identify the leisure trips from other types of trips? (2) is the bike program well connected to the existing public bus services in the city? and (3) how well are the MBS programs providing services to the low-income communities in Topeka?

\subsection{Transportation Type}

For business analysis, determining the type of trips can be very useful for understanding how and why certain trips are generated. In addition, funding from various sources can be used to enrich the customers' experiences and extend the overall lifespan of the MBS system in the city. In this analysis, we use four different criteria (labeled C-\#) to differentiate the "leisure trips" versus transportation trips.

C-1. For the leisure trips, we expect the bike users to complete roundtrips since one-way leisure trips might lead to additional transportation arrangements. In addition, the MBS bikes cannot be held over one hour, thus bike users face the potential risk of losing their access to a shared bike if they take two separate trips to go to a destination and come back to their starting point. Thus, we hypothesize that the leisure trips have their ending locations to be relatively close to their starting locations. In this analysis, we use half a mile (800 meters) as the threshold to define "returning to the same location."

C-2. We also hypothesize that leisure trips would take relatively long durations to achieve the exercise or leisure goal. In this study, we set up the cut-off time for a leisure trip to be over half an hour.

C-3. To exclude weekday commuter trips, we define the leisure trips to start between 10 am and 4 $\mathrm{pm}$, or between $6 \mathrm{pm}$ and $12 \mathrm{am}$ on weekdays.

${ }^{3}$ To request access to Metro Bike Data, see website: https://walton.uark.edu/enterprise/topekametro-bikes.php
C-4. We treat all the trips during the weekends (Saturdays and Sundays) as leisure trips.

In the analysis, we converted the GPX location files to a point feature class and use the ARCGIS "Name" field (trip variable) to construct individual bike trips. We further extracted the Starting and Ending location information for each trip and created two point feature classes to note the starting and ending locations for all the trips (Figure 1).

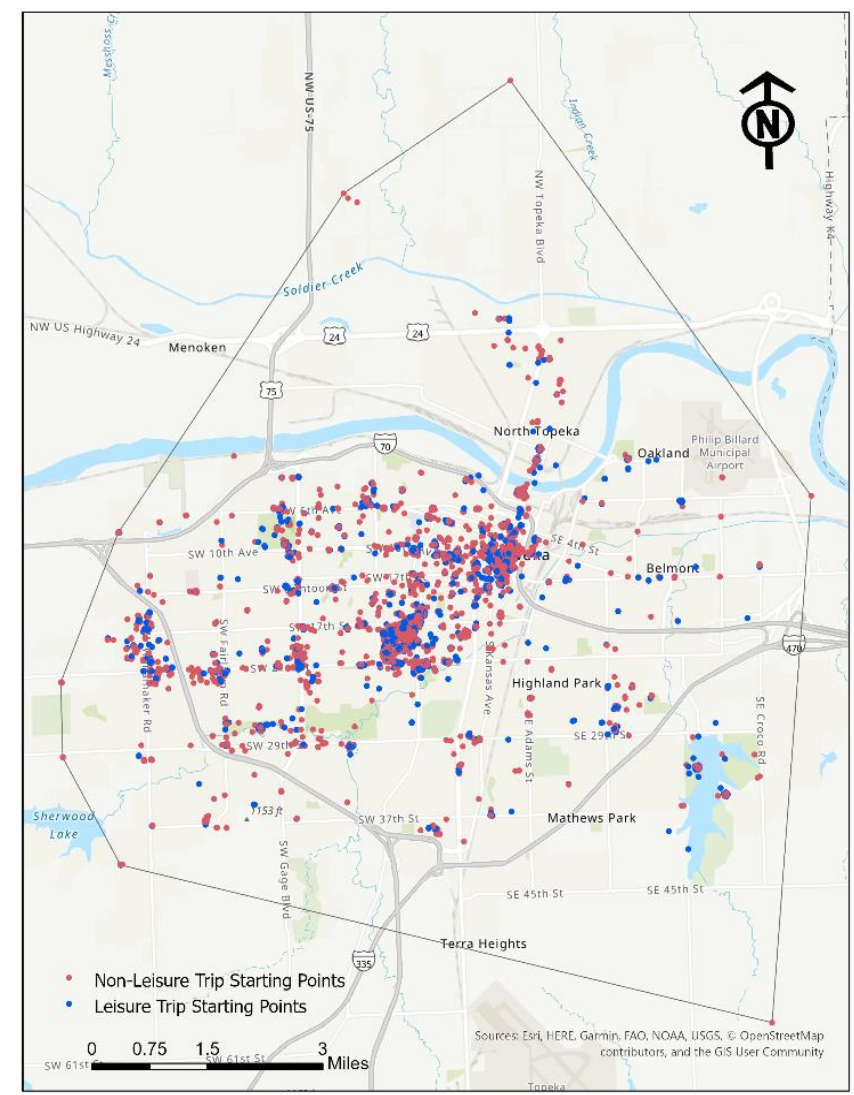

Figure 1 - Leisure vs. Transportation Trips

\subsection{First/Last Mile}

Enhancing the potential for connecting MBS and the existing Metro Bus System would facilitate the First/Last Mile transportation extension for residents. Figure 2 shows a neighborhood example of bus stops, 100-meter buffer zone around bus stops, along with bike hubs and bike stops. The concept behind the First/Last Mile is that users of the bus system are potential users of MBS. Bus system users will take the bus from/to the nearest bus stop, to/from their 
destination/origin. If that destination/origin is still farther than could easily be walked, locating a bike station next to the bus stop could facilitate the bus system users to become MBS users and use the bike to go "the first/last mile". Each bike trip's starting or ending location would be within a certain distance of the bus stop while their ending or starting location should be a certain distance away from the bus stop.

Spatial analysis was used to find trips that fit the two scenarios for the bus/bike users. We believe that for a bus-bike connection to happen, the bike trip should start or end within walking distance from an existing bus stop. In our analysis, we use 100 meters (328 feet), or roughly 1 minute of walking time (based on an average of 3-4 miles/hour of walking speed). Meanwhile, the alternate end or start connection should also happen above a certain distance threshold from the bus stop. We define this distance to be over 400 meters (about 4-5 minutes of walking distance). We assume that these trips are ones that fit the busbike transportation needs. Among the total of 36,355 trips, only $304(0.8 \%)$ are ones that fit our hypothesis. And 189 of the 304 trips (62\%) are ones that START within 100 meters from an existing bus stop (LastMile Connection), while 115 trips are First-Mile Connections. From the map in Figure 3, we can clearly see that the starting locations for Last-Mile trips happen more in the center city, while the starting locations for First-Mile Connections are mostly around the suburbs. And the percentage difference can be explained by the maintenance process implemented by the MB management to collect and relocated bikes that were not at a bike hub. Bikes used as a Last Mile, would be picked up overnight and relocated back to a bike hub. This would eliminate the rider from using the bike as a First Mile the next morning.

Additional insights can be gained by looking at the proximity of all Bike Hubs with respect to the Bus Stops. The relationship between the bus stop locations and the bike hub locations generally confirms that the bike hubs are located close to the existing bus stops (Figure 4). A breakdown of the categories can be recognized in a scaled approach where $74(50 \%)$ of the 148 bike hubs are within 100 meters (328 feet) of an existing bus stop and $114(77.0 \%)$ are within 200 meters (656 feet) of a bus station. Only 9 bike hubs do not have a bus stop within a 500-meter radius. This spatial association is understandable when both

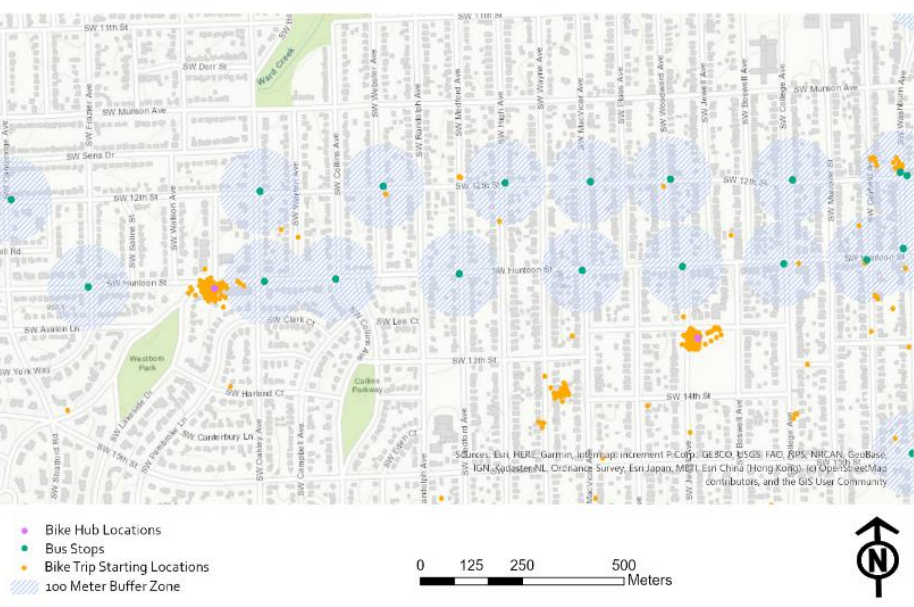

Fiqure 2 - Bike Hub \& Bus Stop Locations

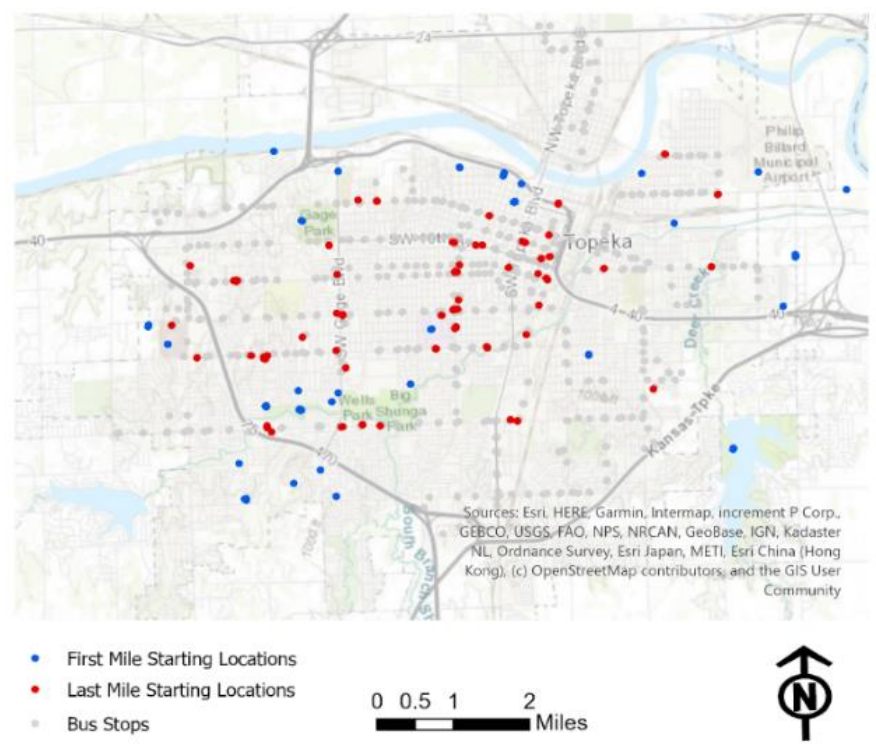

Figure 3 - First/Last Mile Starting Locations

systems are designed to serve a similar user group in

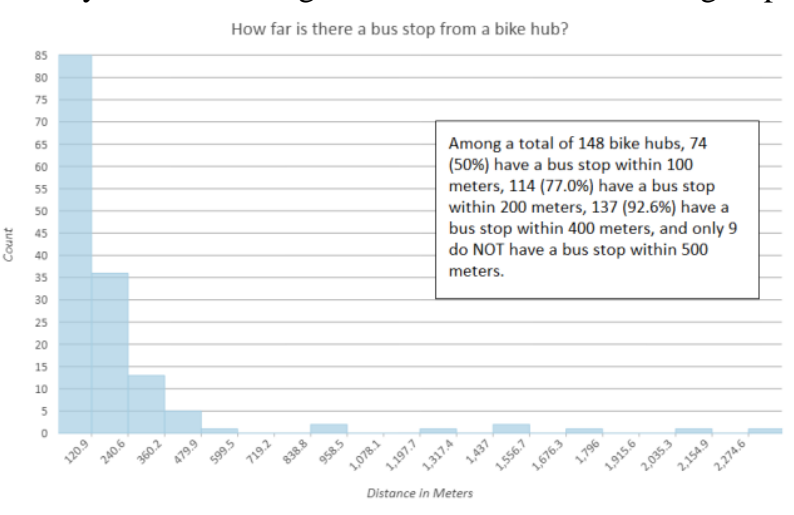

Figure 4 - Bus Stop/Bike Hub Proximity

the city. However, while the bus stops and bike hubs provide an overlapped service, there are areas in 
Topeka where it is lacking an easy access to either the bike or the bus services. This situation limits the bus-bike connection possibility. Additional bike hubs will help link these places with the existing public transportation system, but we also need to look at the socioeconomic characteristics of the bike rides to make sure that the bike hubs be set up in areas that generate the most need for biking services.

To further understand bike usage characteristics, six trip examples were selected to review (Figure 5). All six examples selected meet the bus-bike connection trip criteria (Last-Mile Connection). By adopting the GPS-enabled locational data and the spatial data analysis component from ArcGIS Pro, we can visualize a subset of connection trips that fit our criteria. All the trips shown start from within 100 meters from the bus stop and end more than 400 meters away from the highlighted bus stop. Among the examples, Trip Example 1 and Example 4 overlap for most of the trip, which suggests a repetitive use of the bike service. By further checking the detailed user information of the trips, we found that Trip Example 1-5 are all used by the same user, which suggests how MBS is an important element in some people's everyday lives. Example 6 is a very long trip that did not follow the shortest path and zigzagged through the city, which highlights the flexible use of bikes as a way to finish multiple tasks on the trip.

\subsection{Serving Low-Income Communities}

One of the main goals of the MBS program is to provide community transportation offerings for the benefit of low-income communities. The benefits provided by MBS for low-income communities could not be directly discerned based only on the data collected in the MBS system due to the lack or incompleteness of users' income information. To accommodate this situation, we hypothesize that the aggregated Median Household Income and Per Capita Income around the trip starting locations are good indicators for the bike users' socioeconomic status.

ArcGIS Pro has the ability to make use of U.S. Census data (Open Data) to GeoEnrich each trip starting point. The richness of measuring the goal of serving low-income communities is affected based on the source and aggregation used of the open data. Two sources were used when we created the two column charts: 1) the Median Household Income (MHI) 20142018, and 2) the Per Capita Income (PCI), 2014-2018. In Figure 6 and Figure 7, we aggregated the 2019 Median Household Income and Per Capita Income based on a straight-line half-mile radius from each trip

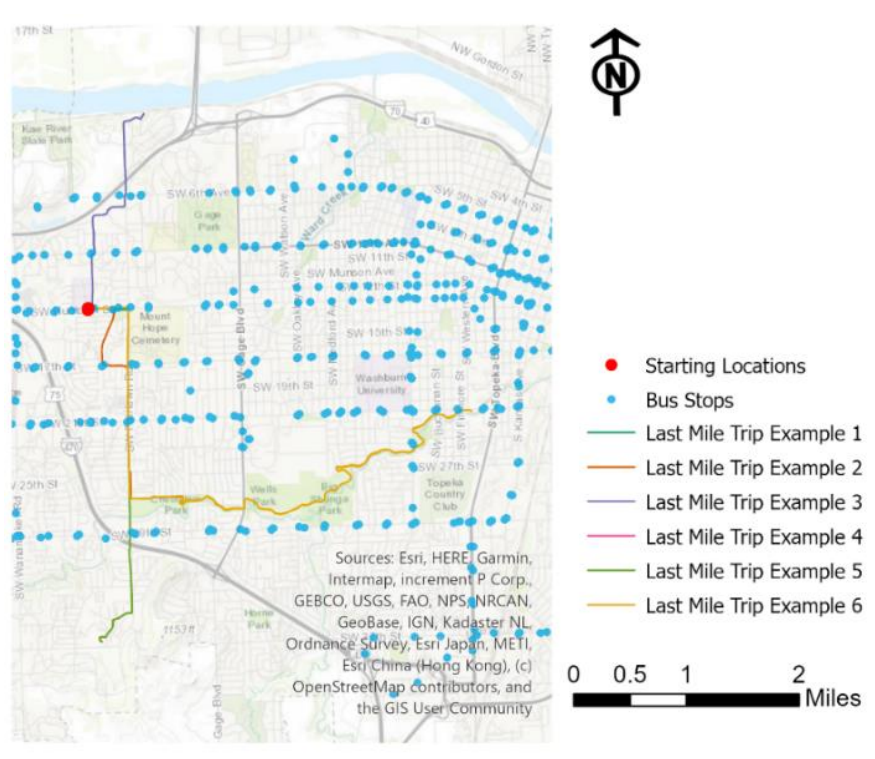

Figure 5 - Last Mile Trip Examples

starting location. The assumption is that the income information at the trip starting point would reflect the users' socioeconomic conditions. The relatively normal distributions of the income information for these two figures provide different views of success with respect to the goal of providing a service to low income communities. Using only 2019 Median Household Income (MHI) data (Figure 6), it appears that about $65.8 \%$ of the bike trips start with a location whose aggregated $\mathrm{MHI}$ is below the average MHI between 2014 and 2018 in the city. While this indicates ridership to be below median income level for the city, it does not clearly reveal usage among the lowest income population. Using the 2019 Per Capita Income (PCI) data (Figure 7), it appears that MBS is achieving a goal as the majority of the trip starting locations (76.4\%) have an aggregated PCI below the average PCI of $\$ 27,145$ in the city. This is not the full story as rider demographics were not available and penetration of bike hubs and bike placement into lowincome housing areas is limited.

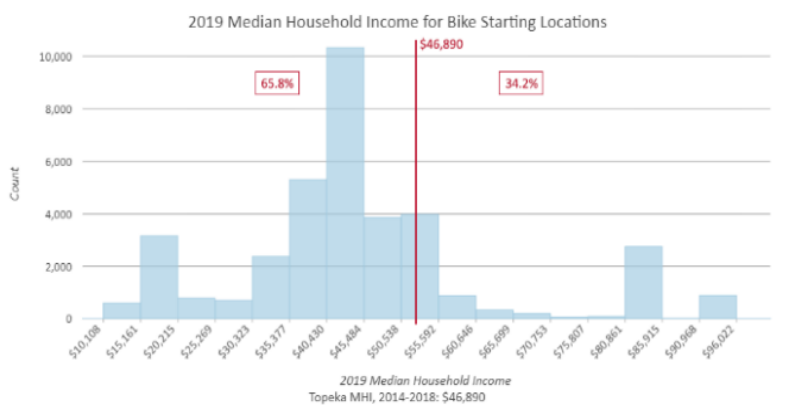

Fiqure 6 - 2019 MHI for Bike Startina Locations 


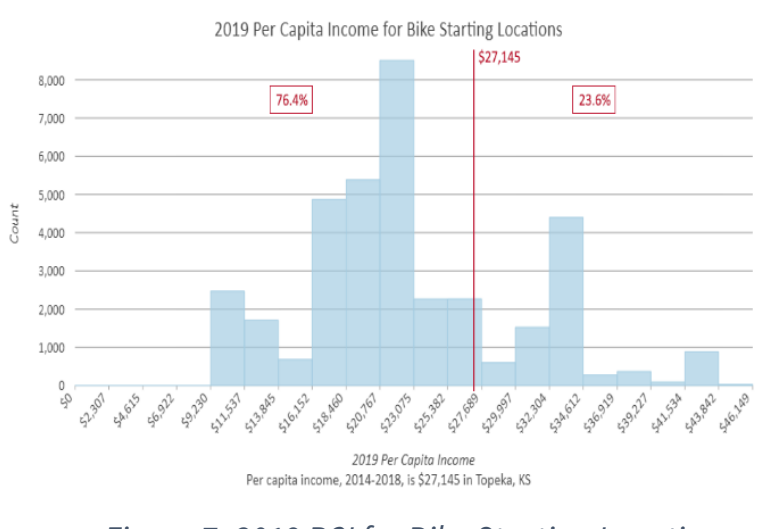

Figure 7-2019 PCI for Bike Starting Locations

Figure 8 shows the relationship between the bike hub usage density and the aggregated MHI for each bike hub location. We calculated the bike hub usage density by calculating the density of trip starting locations within a 500-meter radius for each bike hub location. The higher density indicates the more popular bike hub locations users like to start their trips. We also enrich each bike hub with the Census MHI information. The relationship between the bike hub usage density and the aggregated MHI information is then plotted in a scatter plot. The visualization shows that among the least used bike hubs, the MHI shows a wide distribution. This situation is understandable because these bike hubs are located mostly in the outskirt of the city. The more densely used hubs are located closer to the city center. This observation is supported by the trend line inserted in the scatter graph (Figure 8) showing that the higher the bike usage density, the lower the MHI in the city. So, in general, we argue that the most densely used hubs in MBS are located in the relatively low MHI areas.

Figure 9 illustrates the relationship between the bike hub/bus stop and trip starting locations and the Median Household Income from the American

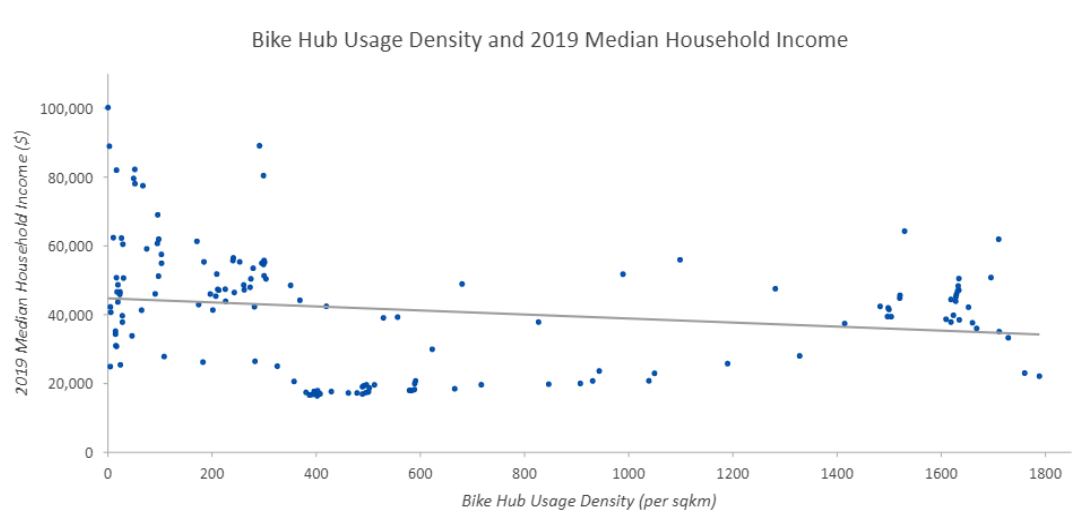

Figure 8 - Bike Hub Usage and Median Household Income
Community Survey (ACS) between 2014 and 2018 [29]. The central city area has relatively low Median Household Income comparing to the outskirt of the city. The bike starting locations (in orange) clearly show a clustering pattern around the central city.

In this case of Enriching MBS's decision making on whether they could achieve a new goal of servicing the lower income community, three separate evaluations are needed to further assess which Enrichment most accurately indicates goal achievement. Those three evaluations are: 1) How MHI vs. PCI differs concerning servicing the lowincome community, 2) Are the time differences for the Enrichment data collection periods (2019 vs. 20142018) affecting this decision, and 3) How accurate is the underlying assumption that trip start point actually represents whether the PCI or the MHI of the bike user? All of these questions represent the richness of system usage by the administrators of the MBS system and require intimate knowledge of each data aspect incorporated into the analysis of goal attainment.

\section{Conclusions}

The contribution of this paper is to address gaps in the transportation segment of the sharing economy and derive business value from GPS data using spatial analysis to enhance management of a bike-share program. Analyzing location data collected by bikeshare information system technology is shown to benefit transit management, as it can be used to gain funding from new supporting organizations. Business decisions regarding operations can integrate different modes of public transportation and service disadvantaged segments of the community. Consistent throughout location analytics is the use of a geographic representation to enhance the decisionmaking capabilities of the user. Benefits can come from augmenting location data, using GeoEnrichment by acquiring public open data to provide additional insights beneficial to making more insightful business decisions. Herein, spatial data analysis with GeoEnrichment using U. S. Census data for socio-economic data pertaining to locations under study has added another important layer of 
information beyond that of routine business information (e.g. membership, purchasing, frequency of use, etc. ...).

\subsection{Insights and Findings}

The locational data and spatial data analysis expand the traditional business analysis to enable spatial location examinations and recommendations. Specifically, this study focused on three business recommendations. 1) Combining the attribute and location inquiry enables differentiating types of bike trips. With 12,328 (33.9\%) of the total rides being classified as "non-transportation" trips, we recommend the MBS program to seek further collaboration with the Department of Recreation to jointly fund MBS. 2) By spatial analysis of the bus-bike connections, we detect few connection rides between the two public service transit systems. The analysis discovers that less than $1 \%$ of the total bike rides fit our criteria to be connection trips, among which, most $(62 \%)$ are last-mile connections. That means $62 \%$ of the connection trips start close to a bus stop and end far away from one. This leads to a recommendation that MBS reduce the current practice of relocating bikes among different hubs at night to move them to "popular" ride starting locations. This current activity not only adds cost to the business, but also may prevent potential "firstmile" trips from happening in the city. (3) This study also examined the potential of MBS to provide services to low-income population in the city. The GeoEnrich function in the ESRI ArcGIS Pro program makes the analysis approachable. The result provides empirical support for the presumption that the users of the MBS have a relatively lower average MHI and PCI in the city.

\subsection{Contributions}

The contributions of our study encompass both a research approach perspective as well as a public sector managerial perspective when using Geolocation data to enhance analysis. Past and potential future organizational goals of bike share programs are assessed by immediate and focused analysis of the data collected from the beginning of the program. Examples of both how the research approach can determine the effectiveness of the goal setting approach of the programs managing directors. These findings may require an iterative approach to operational guidelines in order to further enhance the

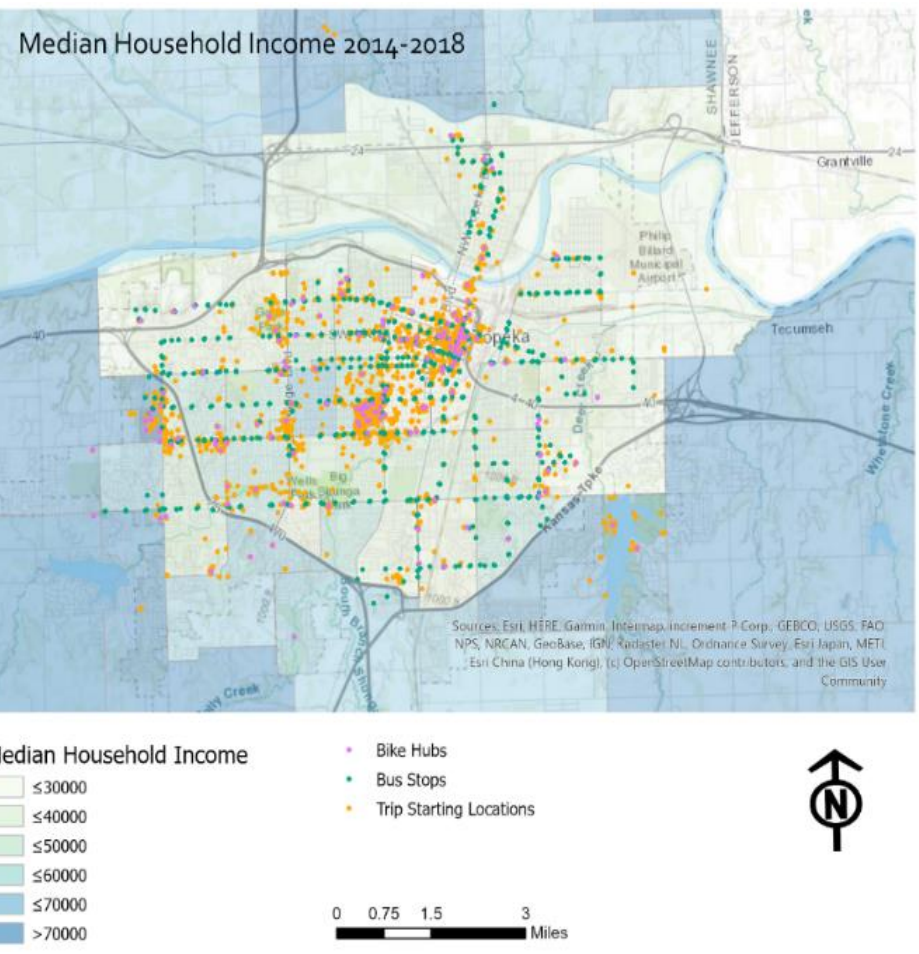

Figure 9 - Median Household Income 2014 - 2018

effective use of the program. Each research question can highlight these methodological contributions.

The first research question provided a series of assumptions on how to identify leisure vs. transportation users. The bike share program was set up with a focus on the enhancement of transportation purposeful options such as rides to work and for errands. The sole funding source for the program has been the city's transportation budget. This analysis provided insights contributed to a rethinking of how the funding sources should be addressed. Specifically, the parks and recreation budget seems appropriate for supporting a portion of the bike share program given strong ridership in leisure areas and for recreation. The research also recognizes that the assumptions of the ride share characteristics should be confirmed. This would necessitate the acquisition of additional data directly from the users as to their rides purpose. The accuracy of this additional data would indicate a level of support for the characteristic assumptions of what a leisure ride looks like.

The second research question concerns the first /last-mile goal of enhancing the transportation system. Less than $1 \%$ of all rides seemed to fit the criteria of first/last mile. In addition, the operational policy of relocating bikes overnight contributed to the imbalance of first mile vs last mile identified rides. 
Finally, the closeness of bus stops to each other and the lack of greater use of bike hubs close to bus stops reduces the users of this categorization of ride types. This part of the analysis offers guidance for creation of a new operational process to better integrate the two transportation systems. An operational policy change is needed to allow more first mile rides. This operational change would be to no longer relocate bikes to hubs overnight. An operational goal of increasing first/last-mile rides would also require a coordination between the bus system (potentially reducing the bus stops) and the bike share (issuance of a bike-bus pass) in order to encourage the first/last mile connection usage of the bikes.

The potential operational goal of supporting the low-income constituents appears to be working as many starting locations are from the relatively lowincome area of the city center. However, this goal rests on the assumption that the bike user's income reflects their starting location. In addition, there are many areas around the city that have relatively few bike hubs or bus stops. In addition, a significance test should be run to determine if the average starting bike trip location is different from the city means. This research provides a location-based analysis approach to assess contributions to various goals of bike share management. These results show operational changes are needed should this community endeavor to address integrating the two transit systems and endeavor to better serve the low-income community.

\section{Limitations}

Goals for the MBS have become better defined and articulated over time, so though the system's initial goals were met, goal-enhancement became of importance once baseline operations were already established. The MBS uses an outside entity for technology and data collection and this limits the operational data collected. This data analysis could only be performed with the data already collected and was performed post hoc. As MB operated a few years before GPS analysis was performed, this analysis would have been more informative and impactful with early implementation. Learnings herein address the gap in literature for addressing funding sources within the sharing economy and should inform future operations for bikeshare programs. Further, this analysis offers approaches to a more integrated analysis of co-located transportation systems and consideration for community demographics.

\section{References}

[1] B. Cohen and J. Kietzmann, "Ride on! Mobility business models for the sharing economy," Organization \& Environment, vol. 27, no. 3, pp. 279-296, 2014.

[2] J. Ursaki and L. Aultman-Hall, "Quantifying the equity of bikeshare access in US cities (No. TRC Report 15-011)," University of Vermont. Transportation Research Center., Burlington, VT, 2015.

[3] E. Fishman, "Bikeshare: A Review of Recent Literature," Transport Reviews, vol. 36, no. 1, pp. 92-113, 2016.

[4] E. Fishman, S. Washington, N. Haworth and A. Watson, "Factors influencing bike share membership: An analysis of Melbourne and Brisbane," Transportation Research Part A: Policy and Practice, vol. 71, pp. 17-30, 2015.

[5] R. Buehler and A. Hamre, "Business and bikeshare user perceptions of the economic benefits of capital bikeshare," Transportation Research Record,, vol. 2520, no. 1, pp. 100-111, 2015.

[6] M. Finger, N. Bert, D. Kupfer, J. J. Montero-Pascual and M. Wolek, "Research for TRAN committee: infrastructure funding challenges in the sharing economy," 2016.

[7] A. Nickkar, S. Banerjee, C. Chavis, I. A. Bhuyan and P. Barnes, "A spatialtemporal gender and land use analysis of bikeshare ridership: The case study of Baltimore City," City, Culture and Society, vol. 18, pp. 1-11, 2019.

[8] M. Ahillen, D. Mateo-Babiano and J. Corcoran, "Dynamics of bike sharing in Washington, DC and Brisbane, Australia: Implications for policy and planning," International Journal of Sustainable Transportation, vol. 10, no. 5, pp. 441-454, 2016.

[9] F. Ogilvie and A. Goodman, "Inequalities in usage of a public bicycle sharing scheme: Socio-demographic predictors of uptake and usage of the London (UK) cycle hire scheme," 
Preventive Medicine, vol. 55, pp. 40-45, 2012.

[10] J. V. Stafford, "Implementing Precision Agriculture in the 21st Century," Journal of Agricultural Engineering Research, vol. 76, p. 267-275, 72000.

[11] C. Palaniswami, P. Gopalasundaram and A. Bhaskaran, "Application of GPS and GIS in Sugarcane Agriculture," Sugar Tech, vol. 13, p. 360-365, 122011.

[12] R. Moen, J. Pastor, Y. Cohen and C. C. Schwartz, "Effects of Moose Movement and Habitat Use on GPS Collar Performance," The Journal of Wildlife Management, vol. 60, p. 659-668, 1996.

[13] E. D. Land, D. B. Shindle, R. J. Kawula, J. F. Benson, M. A. Lotz and D. P. Onorato, "Florida Panther Habitat Selection Analysis of Concurrent GPS and VHF Telemetry Data," The Journal of Wildlife Management, vol. 72, p. 633-639, 2008.

[14] R. A. Morton, M. P. Leach, J. G. Paine and M. A. Cardoza, "Monitoring Beach Changes Using GPS Surveying Techniques," Journal of Coastal Research, vol. 9, p. 702-720, 1993.

[15] F. M. Jacobsen and W. H. Theakstone, "Monitoring glacier changes using a global positioning system in differential mode," Annals of Glaciology, vol. 24, p. 314-319, 1997.

[16] R. Fallourd, O. Harant, E. Trouve, J.M. Nicolas, M. Gay, A. Walpersdorf, J.-L. Mugnier, J. Serafini, D. Rosu, L. Bombrun, G. Vasile, N. Cotte, F. Vernier, F. Tupin, L. Moreau and P. Bolon, "Monitoring Temperate Glacier Displacement by MultiTemporal TerraSAR-X Images and Continuous GPS Measurements," IEEE Journal of Selected Topics in Applied Earth Observations and Remote Sensing, vol. 4, p. 372-386, 62011.

[17] B. DeVries, A. K. Pratihast, J. Verbesselt, L. Kooistra and M. Herold,
"Characterizing Forest Change Using Community-Based Monitoring Data and Landsat Time Series," PLoS ONE, vol. 11, 32016.

[18] A. Habjan, C. Andriopoulos and M. Gotsi, "The Role of GPS-Enabled Information in Transforming Operational Decision Making: an Exploratory Study," European Journal of Information Systems, vol. 23, p. 481-502, 72014.

[19] B. Chaix, J. Méline, S. Duncan, C. Merrien, N. Karusisi, C. Perchoux, A. Lewin, K. Labadi and Y. Kestens, "GPS tracking in neighborhood and health studies: A step forward for environmental exposure assessment, a step backward for causal inference?," Health \& Place, vol. 21, p. 46-51, 52013.

[20] U. S. Census Bureau, "2014-2018 American Community Survey 5-Year Public Use Microdata Samples," 2019. [Online]. Available: https://www.census.gov/programssurveys/acs. [Accessed 11 July 2020].

[21] M. Du and L. Cheng, "Better Understanding the Characteristics and Influential Factors of Different Travel Patterns in Free-Floating Bike Sharing: Evidence from Nanjing, China," Sustainability, vol. 10, no. (4), pp. 1-14, 2018.

[22] U.S. Census Bureau, "2014-2018 American Community Survey 5-year Public Use Microdata Samples," 2019. [Online]. Available: https://www.census.gov/programssurveys/acs.

[23] M. M. Jankowska, J. Schipperijn and J. Kerr, "A Framework For Using GPS Data In Physical Activity And Sedentary Behavior Studies," Exercise and sport sciences reviews, vol. 43, p. 48-56, 12015. 